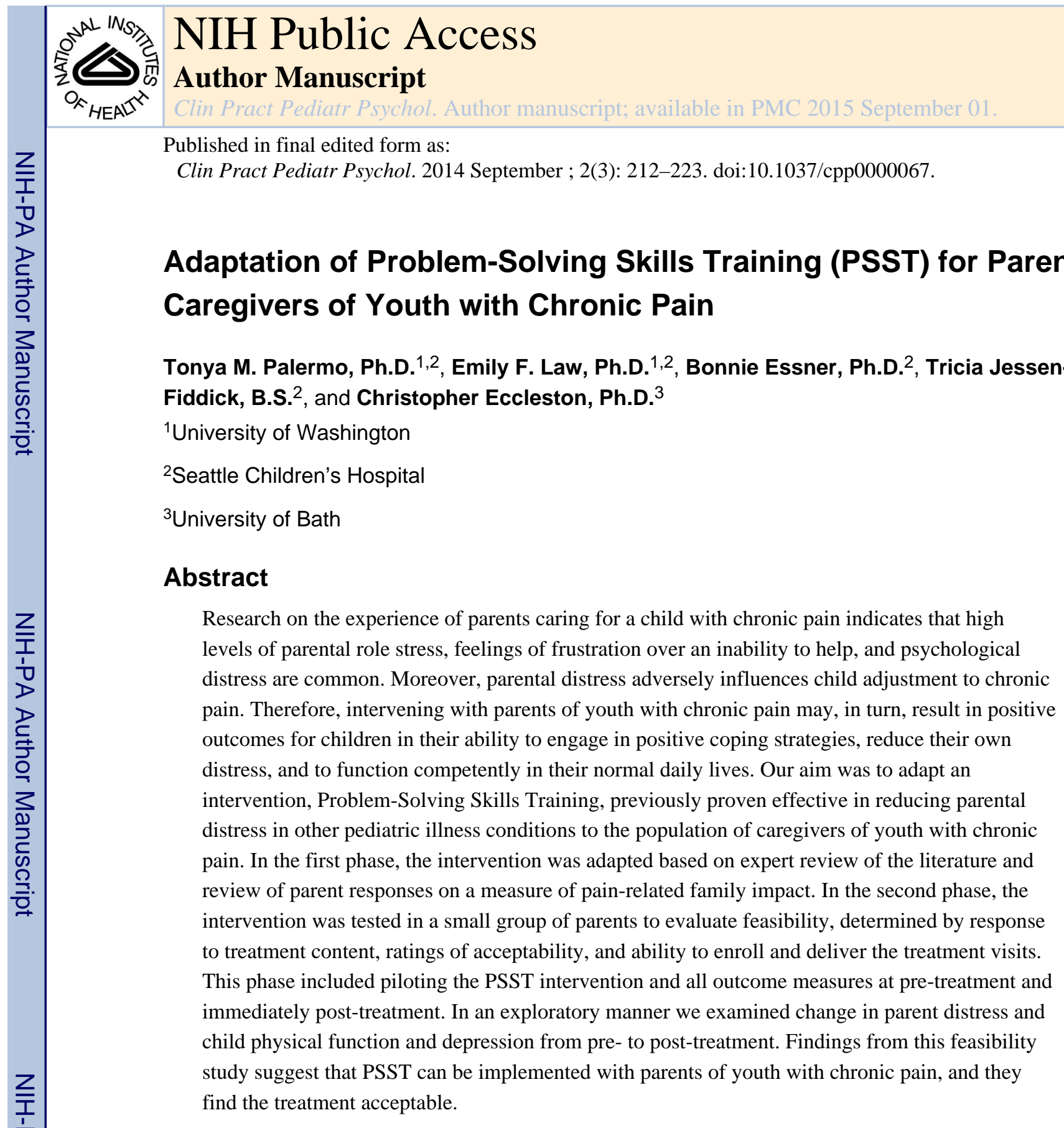

\title{
Keywords
}

pediatric chronic pain; parents; intervention; problem solving therapy; caregiver stress

Conservative estimates suggest that at least $15 \%$ of children and adolescents report chronic pain during childhood, that is, pain lasting up to 3 months (Stanford, Chambers, Biesanz, \& Chen, 2008). A subgroup (i.e., 5-10\% of otherwise healthy youth) report severe pain associated with functional impairment (Huguet \& Miro, 2008). The consequences of chronic pain extend beyond children themselves to potentially include widespread social, relational,

Corresponding author: Tonya M. Palermo, Ph.D., Seattle Children's Research Institute, M/S CW8-6, PO Box 5371, Seattle, WA 98145, USA, 206-884-4208 (tel), 206-985-3562 (fax), tonya.palermo@ seattlechildrens.org. 
emotional and financial impact on parents. In particular, parents report restrictions in their own lives, unwelcome dependency, marital and financial difficulties, and feelings of hopelessness (Hunfeld et al., 2002). In a clinical sample of youth with chronic pain, Eccleston, Crombez, Scotford, Clinch \& Connell (2004) found that average levels of parental role stress were high, with $31 \%$ of parents reporting clinically significant distress. Moreover, anxiety and depressive symptoms were commonly reported by parents (Eccleston, et al., 2004). Similarly, Jordan et al. (2008) found a negative impact of chronic pain on maternal social and emotional functioning. In one of the few qualitative studies in this area, Jordan, Eccleston, and Osborn (2007) interviewed 17 parents of youth with chronic pain about their experience, finding that parents reported struggling for control over their lives, experienced considerable strain at being unable to help their child in pain, and judged their lives fundamentally changed.

There has been a renewed research focus on the broader contextual factors relevant to the management of pediatric chronic pain. Parent and family factors, in particular, have been identified as important in understanding the context in which pain treatment is provided. Palermo and Chambers (2005) proposed a conceptual model of a bidirectional relationship between parent and family factors (e.g., parental responses to pain behavior; family environment) and children's pain experience. In this model, parent and family factors are hypothesized to increase the risk for pain and disability, and in turn, pain and disability are expected to impact parent and family life. Parental distress is considered as a family level variable where the child's response to pain is influenced under the conditions of the family or parent's overall functioning. Therefore, interventions applied to parent caregivers for the purpose of reducing distress, anxiety, and depressive symptoms would be hypothesized to have downstream effects on improving child experiences with chronic pain.

There is an emerging literature on the association between parental functioning and child adjustment to chronic pain. For example, an early study in children with juvenile rheumatoid arthritis found that greater emotional distress of the mother was related to higher levels of child reported pain (Ross et al., 1993). Similarly, higher levels of parental psychological distress (Logan \& Scharff, 2005) and less healthy family functioning (Lewandowski, Palermo, Stinson, Handley, \& Chambers, 2010; Palermo, Putnam, Armstrong, \& Daily, 2007) have been associated with greater pain-related disability in youth.

In the most recent systematic review of the efficacy of psychological therapies for pediatric chronic pain (Fisher et al., in press), psychological treatment was found to be effective in reducing children's pain and disability; however, there was variability in the size of effects between pain conditions and positive benefits were not found for reduction in child depression or anxiety. These trials used a range of cognitive-behavioral intervention strategies (e.g., relaxation skills training, cognitive strategies, guided imagery, exposure and acceptance, and parent operant strategies). Parent operant interventions included instruction to parents to modify their own parenting behavior through minimizing response to pain complaints and encouraging adaptive behavior (Kashikar-Zuck, Swain, Jones, \& Graham, 2005; Sanders, Shepherd, Cleghorn, \& Woolford, 1994). However, at present, no intervention has been developed specifically to treat parental distress in the context of their 
child's chronic pain. There is a critical need to consider methods of reducing parental distress in order to enhance children's adjustment to chronic pain.

As highlighted by Palermo and Eccleston (2009), in the cognate literature on parental and caregiver adjustment to other serious pediatric health conditions (e.g., cancer, traumatic brain injury), there has been development and testing of interventions to decrease parental distress. In a Cochrane review on this topic, problem-solving skills training (PSST) delivered to parents of children with chronic conditions was found to be effective in reducing the distress (improving parental mental health and behavior) associated with parenting a child with a chronic illness (Eccleston, Palermo, Fisher, \& Law, 2012). In contrast, there was no evidence for the effectiveness of other cognitive and behavioral therapies in improving parental mental health or behavioral outcomes. Similarly in an updated systematic review and meta-analysis of parent interventions (Law, et al., in press), PSST was found to improve parent mental health and behavior at post-treatment and followup in several additional chronic medical conditions.

PSST is based on the social problem-solving model developed by D'Zurilla and Nezu (1999, 2007) in which problem solving skills are taught using modeling, behavioral rehearsal, and performance feedback. Typically, PSST involves instruction in several steps of problem solving which may include adopting a positive problem orientation, problem definition and formulation, generating alternative solutions, evaluating options, implementing solutions, and evaluating the solution performance. Homework practice involving opportunities to solve identified problems is an important part of the training. Efficacy of PSST has been evaluated in caregivers of both adult and pediatric medical populations, gaining considerable empirical support (Grant, Elliott, Weaver, Bartolucci, \& Giger, 2002; Rivera, Elliott, Berry, \& Grant, 2008; Sahler et al., 2005; Sahler et al., 2002; Seid, Varni, Gidwani, Gelhard, \& Slymen, 2010; Wade, Carey, \& Wolfe, 2006). One of the first research teams to use PSST in caregivers of children with health problems was Sahler and colleagues (2002). These investigators have examined the feasibility and efficacy of PSST for reducing distress in mothers of children with newly diagnosed cancer (Sahler, et al., 2005; Sahler, et al., 2002). Findings demonstrated significant improvements in problem-solving skills and significant reduction in anxiety and depressive symptoms among mothers who received an eightsession PSST intervention when compared with mothers who received usual psychosocial care (Sahler, et al., 2005).

Several other studies have been conducted using problem solving therapy interventions with caregivers in distress. Kazdin and Whitley (2003) evaluated parental problem-solving therapy to enhance therapeutic change in children with aggressive and antisocial behavior. They found that parental problem-solving led to reduced stress and depressive symptoms in parents and greater therapeutic change in behavior for children. Wade and colleagues (2006) used problem solving therapy successfully in Internet-based sessions to reduce distress in parental caregivers and to promote positive changes in social behavior in youth with traumatic brain injury. Similarly, Rivera et al. (2008) used problem solving therapy in a sample of caregivers of adults with traumatic brain injury using a combination of in-home visits and telephone calls to deliver treatment, finding significant reductions in depression and health complaints, and significant improvements in problem-solving skills post- 
treatment. Although parent outcomes have been primary in the majority of trials of problem solving interventions (e.g., Sahler, et al., 2005), there is emerging evidence that child outcomes may also be impacted by intervention.

Because the evidence base for parent interventions indicated that problem solving therapy and specifically PSST was a promising treatment, we aimed to adapt this intervention to the population of parent caregivers of youth with chronic pain. The aim of the present study was to develop and evaluate feasibility and acceptability of PSST as an intervention to reduce distress in parent caregivers of youth with chronic pain, with a focus on whether a full trial could be successfully performed. We report two phases of study to 1) adapt and develop the intervention, and 2) pilot the intervention in a small group of parents to evaluate feasibility, determined by response to treatment content, ratings of acceptability, and ability to enroll and deliver treatment visits.

We hypothesized that, similar to work with PSST in parent caregivers of children with other chronic health conditions, parent caregivers would engage in treatment as demonstrated by participation in intervention visits and completion of homework assignments. Further, we expected that parents would rate PSST as acceptable and be satisfied with PSST intervention. We also hypothesized that therapists would report that parents were receptive to treatment, that they established good rapport, and understood the PSST process. Last, we explored the feasibility of administering our outcome measurement protocol and examined parent and child pre-post change scores on outcome measures.

\section{Methods and Results}

\section{Phase 1: Adaptation of therapy materials}

This study was approved by the Institutional Review Boards at both study sites. The first phase of the study involved adaptation of therapy materials from a brief, manualized skillsbased PSST intervention developed for caregivers of children with cancer by Sahler and colleagues ${ }^{1}$ (2002). The research team, which consisted of clinical pediatric psychologists with expertise in pain, cognitive-behavioral, and family therapies, reviewed these existing materials and relevant published literature on problem solving therapy (D'Zurilla \& Nezu, $1999,2007)$ to develop therapist and parent treatment manuals.

We retained the overall philosophy and steps of the PSST program developed by Sahler and colleagues (2002), including the acronym "Bright IDEAS" and the logo of a lighted bulb where "Bright" represents the sense of optimism (positive orientation) about solving problems. The letters I (Identify the problem), D (Determine the options), E (Evaluate options and choose the best), A (Act), and S (See if it worked) indicate the five steps of problem-solving as originally developed by D'Zurilla and Nezu (1999) and reinterpreted by the Sahler group (2002).

${ }^{1}$ We received permission from Dr. O.J. Sahler to use and adapt for use the materials developed for the Maternal Problem-Solving Skills Training Project that were copyrighted by her in 2002. 
Treatment materials were modified through the addition of examples specific to parents of children with chronic pain. In order to obtain parent-generated examples, the team reviewed data from an ongoing study that included a measure of parental impact, the Bath Adolescent Pain - Parental Impact Questionnaire (Eccleston et al., 2005) in 95 parents of youth with chronic pain. Parents completing this measure provided open-ended responses to the item "Please tell us about anything else you feel is important for us to know about how caring for a young person with chronic pain impacts on your life."

From these responses, the team developed a list of common problems and issues faced by parents of children with chronic pain to use in a worksheet called Problems to be Solved. The purpose of this worksheet was to help parents generate a list of problems to address during the treatment. Compared to the type of examples of problems used in the Sahler et al (2002) treatment materials with mothers of children with newly diagnosed cancer, the problems generated by parent caregivers of youth with chronic pain were of longer-term duration, did not tend to reflect acute distress, and were focused primarily on issues in the home (rather than in the hospital). Initial versions of therapy manuals were reviewed and edited by the research team. A two-day training workshop was held to train three therapists in PSST. Following this training, several additional modifications were made to the therapy manuals, including the creation of a brochure for parents which illustrated the steps of the problem solving process using vignettes of families of children with chronic pain. Our final set of instructional materials included a therapist manual, a parent treatment manual, parent brochure, and problem-solving worksheets.

\section{Phase 2: Pilot intervention}

The second phase of the study involved piloting the PSST intervention with parents of youth with chronic pain to examine feasibility of the therapy and of the study protocol.

Participants-Participants were recruited from pediatric pain specialty clinics at two sites in the Pacific Northwest. Each site's Institutional Review Board approved the study prior to onset of study procedures. Inclusion criteria included: (a) child age between 10 and 17 years, (b) child had pain present for at least 3 months duration which occurred at least once/week and interfered with daily functioning, (c) child received specialty evaluation and treatment for an idiopathic pain condition, and (d) parents were English-speaking. Exclusion criteria included: (a) child had a diagnosis of a comorbid serious health condition (e.g., arthritis, diabetes), (b) child's pain had been present for less than 3 months, (c) parent had lived with the child for less than one year, and (d) parent had active psychosis or suicidal ideation.

Participants in the pilot intervention were 6 mothers ( $M_{\text {age }} 45.6$ years) of youth ( $M_{\text {age }} 14.5$ years) with chronic musculoskeletal, abdominal, and headache pain who had been evaluated and treated for their chronic pain condition in one of two pediatric pain clinics. See Table 1 for additional descriptive information about the sample.

Parents completed standardized measures of depression, mood disturbance, pain catastrophizing, parenting stress, miscarried helping, problem-solving skills, and impact of chronic pain at pre-treatment and immediately post-treatment. They also completed a treatment satisfaction measure at the end of the intervention. Children completed a measure 
of physical function and depressive symptoms at pre-treatment and immediately posttreatment.

Parental Problem-Solving Skills Training (PSST)—Sessions were conducted according to the detailed therapy protocol adapted for this study. The PSST intervention was developed to be delivered in 6 to 8 individual treatment sessions ( 1 hour each) with the parent over approximately 8 weeks. In collaboration with the parent, therapists determined when treatment should terminate (at the 6-8 session point) based on progress of the caregiver with learning PSST. Each parent was given a verbal overview of the intervention, the parents' manual, and the parent brochure. At the end of each session, parents were given a homework assignment related to identifying and solving a problem of immediate interest to them. The Problems to be Solved worksheet, consisting of a checklist of common problems experienced by parents of children with chronic pain, was used to help parents identify relevant problems to work on in session. Their problem-solving efforts were reviewed at each subsequent session.

An overview of the goals and content of treatment sessions is provided in Table 2. In the first session, therapists focused on establishing rapport and introducing parents to the PSST program. The therapist used a non-directive style to allow parents to tell their personal stories about how their child's chronic pain impacts the family. Session 2 was intended to provide the caregiver with instruction in learned optimism and problem orientation. Therapists reviewed the Bright Ideas Wheel, highlighting that learned optimism is in the center of the wheel. Caregivers were instructed in identifying automatic thoughts and feelings, generating self-statements, and approaches to positive problem orientation (e.g., belief that problems can be solved). During the next session, therapists provided instruction in each of the problem solving steps. Variability in the amount of time needed to accomplish this was built into the treatment program. The goal was to teach all of the problem solving steps by the end of the third session. However, ample time was allotted to continue to review problem solving steps in subsequent sessions. Parents identified any problem that they wished to work on using the Problems to be Solved worksheet.

The therapist used several cognitive-behavioral strategies to teach problem solving skills to parents including modeling, behavioral rehearsal, performance feedback, and generalization of skills. For example, the therapist provided direct examples of problem-solving components to the parent and in vivo practice opportunities in session where the caregiver could rehearse or practice the specific components of the problem-solving process using an illustrative situation during the session. Structured homework assignments were given in each session to provide opportunities for the parent to try the new problem-solving skills. During sessions 4-6, the therapist taught solution verification and assisted the parent in continuing to practice the problem solving steps by working through the same problem or new problems. Sessions 6-8 were focused on relapse prevention and consolidation of skills. The therapist collaborated with the caregiver to determine when treatment would end, once all problem solving skills had been successfully learned. Therapists emphasized using emotional distress as a cue to identify and engage in problem solving efforts in the future and supported caregivers in their independent ability to carry out problem solving on their own. 
Therapist Qualifications and Training-Three therapists were trained to deliver PSST for the pilot intervention. Two therapists were psychology trainees (one intern and one postdoctoral fellow) and one therapist was a licensed psychologist. Therapists had experience in cognitive-behavioral therapy and in pediatric chronic pain management. They participated in the 2-day training workshop to learn the components of PSST and to practice and role-play therapy components. Ongoing supervision during the pilot intervention occurred in weekly hour-long supervision meetings with the first author.

\section{Measures}

Demographics and Pain Characteristics-Parents completed a background form to identify the child and parent's ethnicity, parental marital status, parental educational attainment, parental age, parental employment, and family income. Children rated their usual pain intensity using an 11-point Numerical Rating Scale (NRS) ranging from 0 (no pain) to 10 (worst pain possible).

Parent Problem Solving Skills-Parents completed the Social Problem Solving Inventory-Revised (SPSI-R), a 52-item self-report instrument linked to a five-dimensional model of social problem-solving (D'Zurilla, Nezu, \& Maydeu-Olivares, 2002). The SPSI-R consists of five sub-scales that measure two different problem orientation dimensions (Positive and Negative) and three different problem-solving proper dimensions (Irrational Problem-Solving, Impulsivity/Carelessness Style, and Avoidance Style). These subscales are combined to form a total problem-solving skills score. All scores are converted to Standard Scores, with higher scores indicative of greater problem-solving ability. The SPSI-R has strong reliability and validity estimates, and was used in previous caregiver PSST studies (Sahler, et al., 2005).

Parent Depressive Symptoms and Negative Affectivity-Parents completed the Beck Depression Inventory (BDI-II), a 21-item self-report measure that assesses the cognitive, affective, and behavioral components of depression. Higher scores reflect more depressive symptoms. The BDI-II is widely used for both clinical and research purposes, and has demonstrated strong reliability and validity. The BDI-II has been used in a number of studies assessing the relationship between problem-solving ability and depression (Morley, Williams, \& Black, 2002).

Parents completed the Profile of Mood States (POMS-Brief Form) as an additional measure of negative affectivity (McNair, Lorr, \& Droppleman, 1992). The brief form includes 30items that ask about feelings over the previous week. Six affective states are assessed: tension-anxiety, depression-dejection, anger-hostility, vigor-activity, fatigue-inertia, and confusion-bewilderment. These subscales are combined to form a total POMS mood disturbance score. All scores are converted to T-scores; higher scores reflect a more intense mood rating for the respective emotional domain. Internal consistency, test-retest reliability and validity are excellent (McNair et al., 1992).

Parent Role Stress-Parents completed the Parenting Stress Index-Short Form (PSI-SF), a 36-item questionnaire that assesses parental distress, parent-child dysfunctional 
interactions, and difficulty of parenting the child (Abidin, 1995). These subscales are combined to generate a total stress score. Scores are transformed into percentiles, with higher scores indicating greater parenting stress. This measure displays good construct validity, test-retest stability and predictive validity (Haskett, Ahern, Ward, \& Allaire, 2006).

Parent Miscarried Helping-Miscarried helping was originally conceptualized by Anderson and Coyne (1991) as a way of understanding how interpersonal conflict develops in families of children with chronic medical conditions. Parents completed the Helping for Health Index (HHI), a 15-item questionnaire originally designed to assess miscarried helping among parents of youth with diabetes, and has demonstrated good reliability and validity in that population (Harris et al., 2008). For this study, an adapted version of the HHI published for children with chronic pain was used (Fales, et al., 2014). A total score is calculated by summing across all items with higher scores indicating greater miscarried helping.

Parent Pain-Related Catastrophic Thinking-Parents completed the Pain Catastrophizing Scale for Parents (PCS-P) (Goubert, Eccleston, Vervoort, Jordan, \& Crombez, 2006) to assess their catastrophic thinking related to their child's pain. The PCS-P is a 13-item adaptation of the Pain Catastrophizing Scale for Children (Crombez et al., 2003). Respondents rate the frequency at which they experience certain thoughts and feelings about their child's pain. Higher scores are indicative of more frequent catastrophic thinking. The PCS-P has demonstrated adequate reliability and validity for youth with chronic pain (Goubert et al., 2006).

Parent Impact of Pediatric Chronic Pain-The Bath Adolescent Pain-Parental Impact Questionnaire (BAP-PIQ) (Jordan, Eccleston, McCracken, Connell, \& Clinch, 2008) was completed by parents. This 62 -item measure was developed to assess changes in functioning and behavior associated with parenting an adolescent with chronic pain. Higher scores indicate more impaired functioning. The 11-item parental behavior subscale was used for this study. The parental behavior subscale measures the degree to which parents use avoidance and engagement strategies to interact with their children when they are in pain, and has demonstrated adequate reliability and validity (Jordan et al., 2008).

Child Pain-Specific Physical and Emotional Functioning-Children completed the Bath Adolescent Pain Questionnaire (BAPQ, Child Version) to measure their pain-specific physical and emotional functioning (Eccleston, et al., 2005). The BAPQ contains 61 items that load onto seven subscales: social functioning, physical functioning, depression, general anxiety, pain-specific anxiety, family functioning, and development. The 9-item physical functioning and 6-item depression subscales were used in this study. The physical functioning and depression subscales have demonstrated adequate reliability and validity for youth with chronic pain (Eccleston et al., 2005).

Treatment Satisfaction-Parents completed a modified version of the Treatment Evaluation Inventory-Short Form (Kelley, Heffer, Gresham, \& Elliot, 1989). This is a 9-item scale regarding satisfaction with the therapeutic process during the course of treatment and satisfaction with the outcome of therapy, rated on a 5-point scale from $1=$ strongly disagree 
to $5=$ strongly agree. This measure has demonstrated good reliability and validity (Kelley et al., 1989).

Therapist Records-We collected feasibility data including scheduling information (e.g., show rate, rescheduled visits), therapist records, and session worksheets. Each session, therapists rated parents' motivation to learn, receptivity to learning, understanding of the therapy process, completion of homework, and rapport using 0-10 scales $(0=l o w, 10=$ high). Therapist records were completed at the end of each session.

\section{Results of the Pilot Intervention}

Engagement-Each parent received 3 to 7 intervention visits $(M$ sessions $=4.5)$. Parents were adherent to scheduled visits, with few missed sessions (range 0-1 per participant) and few rescheduled visits (range 0-3 per participants). The decision to terminate treatment was made collaboratively between parents and therapists based on receiving all treatment components and demonstrating the ability to use the problem solving skills independently. One caregiver terminated treatment early (after 3 sessions) because her child no longer required treatment at the pain clinic and she did not want to return separately for continued study visits. All parents were offered the option of completing sessions by telephone, although use of telephone sessions was rare ( 2 of 27 sessions). Completion of betweensession homework assignments was high, with therapists on average rating parents as being compliant with homework completion $(M=8.4 / 10)$.

Satisfaction and Acceptability-Therapist-reported ratings indicated that parents were highly motivated $(M=9.5 / 10)$, receptive to learning $(M=9.4 / 10)$, understood the PSST process $(M=8.6 / 10)$ and established strong rapport $(M=9.0 / 10)$. Parents reported a high degree of satisfaction with the intervention $(M=36.5 / 45)$ and that they found it to be an acceptable treatment for their child's chronic pain $(M=4.5 / 5)$.

Pilot Outcomes-Five parent-adolescent dyads completed the pre-treatment assessment and four dyads completed the post-treatment assessment. Parents and adolescents demonstrated positive change in all outcome measures from pre- to post-treatment (see Table 3). From pre- to post-treatment, parental problem-solving skills improved ( $M=100.6$ to 113.3, respectively), parenting stress decreased ( $M=90.8$ to 55.0), depressive symptoms decreased ( $M=14.0$ to 3.0), mood disturbance declined ( $M=54.0$ to 48.8), parent-reported miscarried helping decreased $(M=37.0$ to 28.8$)$, parents' catastrophic thinking about their child's pain declined ( $M=36.8$ to 25.5 ), and parents' maladaptive behavioral responses to their child's pain also declined $(M=20.6$ to 16.3$)$.

Adolescents reported concurrent improvements in their own physical functioning $(M=11.0$ to 6.8$)$ and depressive symptoms $(M=15.8$ to 11.5$)$ from pre- to post-treatment.

\section{Discussion}

The aim of the present study was to adapt a successful problem solving intervention to the unique needs of parents of youth with chronic pain. Adaptation of the treatment materials was informed by qualitative data from parents regarding their experience of parenting a 
child with chronic pain and the impact of their child's pain condition on their daily life. Pain presents unique challenges for parents because there is often uncertainty about diagnosis and treatment options. Furthermore, these youth have typically experienced pain for many months or years prior to establishing care in a specialized pediatric pain clinic. Thus, adapting PSST required a focus on the chronicity of problems encountered by parents. As part of this process, we developed a list of common problems generated from a parent impact measure to help parents identify problems that they wanted to target in treatment. Anecdotally, this list was an important treatment tool during pilot testing. Parents often initially reported that they had few problems or that they already had a good deal of expertise in solving problems due to the longstanding nature of their child's illness. Reviewing common problems experienced by other parents of children with chronic pain normalized these challenges and assisted parents in selecting problems that they wanted to address in treatment.

We also conducted a pilot test of the intervention with a small group of parents of children with chronic pain to evaluate feasibility and acceptability of the treatment and outcome assessment protocol. Pilot testing demonstrated that parents could be recruited, retained, and complete the treatment protocol. Parents reported a high degree of treatment satisfaction while successfully learning and applying PSST skills. Therapists reported that parents were receptive to treatment, that they established good rapport, and understood the PSST process. Parents were adherent to scheduled sessions, with a low rate of no-shows and rescheduled appointments. Completion of homework assignments was also high. This high level of feasibility is similar to studies of PSST with parent caregivers of youth with other medical conditions (e.g., Sahler, et al., 2005).

Preliminary examination of pre- to post-treatment change on parent and child outcome measures showed that parents remain in treatment, and comply with completing both the treatment and the assessment/evaluation methods. Results from the pilot participant dyads were all in the direction of improvement in outcomes following PSST. Inferential statistics were not undertaken as this was outside the limits of this investigation. However, the change in measures can be interpreted as limited evidence of their reactivity to treatment and hence support their value for use in a larger trial. Further, speculatively, the data add credence to the original hypothesis that PSST may be a promising treatment for reducing distress among parents of children with chronic pain. Treatment that targets parent distress may lead to improvements in child functioning and mood outcomes.

The results of both phases show that a full trial is feasible. We have now begun a full randomized controlled trial (ClinicalTrials.gov identifier: NCT01496378). We aim to recruit 60 parents randomized to two conditions (PSST versus usual care). The study is powered to find a large effect (SMD of 0.83) on the primary outcome of parent depressive symptoms. In addition to the primary hypothesis of a large effect of PSST on parent depressive symptoms, we also have secondary hypotheses that PSST will produce positive change in parenting stress, problem-solving skills, and child physical and emotional functioning in comparison to usual care. 
Results of this pilot study led us to further tailor the PSST intervention for the RCT. For example, therapist ratings indicated that parents of children with chronic pain demonstrated mastery of the problem solving skills within 4 to 6 sessions. Therefore, treatment duration for our ongoing RCT of PSST was modified to be delivered in 4 to 6 sessions. One parent in the pilot study terminated treatment early (after 3 sessions) due to lack of interest in returning for visits after her child completed treatment in pain clinic. We did offer telephone sessions during pilot testing and, although there was limited use, we decided to continue to offer telephone sessions in the ongoing RCT in order to enhance treatment retention and further test feasibility of this approach. PSST has been delivered successfully to caregivers of other medical populations via remote modalities such as the telephone, with equivalent effects on caregiver outcomes compared to face-to-face treatment (Rivera, et al., 2008).

Results also indicated that changes were needed to improve the feasibility of our outcome assessment procedures. Outcome assessments were completed by $5 / 6$ parent-child dyads at pre-treatment and 4/6 dyads at post-treatment. Incomplete outcome assessments were due to a variety of factors including communication challenges in tracking outcome assessments at the two sites, variability in administration of assessments in person vs. via postal mail, and initiating treatment as soon as participants informed us that their completed assessment packet was in the mail. For the ongoing RCT, we now require tracking of assessment administration by a single research assistant, completion of all assessments via postal mail, and receipt of baseline assessments prior to randomization.

\section{Limitations}

Given that $100 \%$ of participants were mothers, our results are reflective of mother's responses to PSST and not fathers. This is a common limitation in pediatric psychology research and likely reflects typical gender roles in caring for children with chronic health conditions. Our sample is also predominantly Caucasian, college-educated, and middleclass. Although these demographic characteristics are similar to other published studies conducted in pediatric pain clinics, it will be important to consider potential generalizability to more diverse populations in the future. It is also important to note that this study was limited to a parent problem solving skills training intervention only. Research is needed to develop and test the feasibility of combined parent and child interventions targeting parent distress and child pain coping skills. Finally, our pilot intervention was designed to examine feasibility and acceptability of treatment only and was not designed to evaluate efficacy of PSST. Efficacy of PSST for parent caregivers of youth with chronic pain will be addressed in our ongoing, multi-site RCT comparing PSST to a usual care condition.

\section{Clinical Implications and Future Directions}

Parents of youth with chronic pain are known to experience considerable parenting stress and negative affectivity, similar to the other caregiver populations in which PSST has been evaluated. Consequently, an intervention to reduce stress and negative affectivity in parents of youth with chronic pain is relevant and expected to have a high impact in this population. Focusing on interventions designed to reduce parental distress introduces a new conceptualization to family and parent factors associated with pediatric chronic pain that is 
consistent with an integrative model of operant behavioral and family systems theory (Palermo \& Chambers, 2005).

Because parent-only behavioral interventions are not currently a part of standard care in pediatric pain clinics, incorporating PSST for parent caregivers of youth with chronic pain into clinical practice may raise several challenges. First, clinicians will need to determine whether separate providers are needed to deliver treatment to parents and children or if a single provider will treat all family members. If multiple providers are working with a single family, steps will need to be taken to facilitate care coordination between providers. Second, there may be billing concerns that arise when delivering treatment only to parents particularly if the child does not accompany the parent to clinic. Third, clinicians may want to consider remote modes of treatment delivery, such as telephone or the Internet, to facilitate access to care for parents with limited time or financial resources or for those parents who do not live near a pediatric pain clinic. These types of systems issues will need to be addressed in order to facilitate delivery of parent-based interventions within pediatric pain clinics.

\section{Conclusions}

Parenting a child with chronic pain is a challenging and complex experience. Parents play an important role in their child's ability to adapt to chronic pain and function in daily life. Currently, strategies are needed to address the emotional functioning of parents of children with chronic pain. PSST has demonstrated efficacy for reducing distress among caregivers of children and adults with a variety of medical problems. Results from this study suggest that PSST is a feasible and acceptable intervention for parents of children with chronic pain. PSST may be a promising treatment for improving parent distress and child physical and emotional functioning in families of children with chronic pain.

\section{Acknowledgments}

We acknowledge the guidance provided by Dr. Robert Butler in adapting the problem solving skills training material.

Funding: Supported by R21HD065180 from the Eunice Kennedy Shriver National Institute of Child Health and Human Development (TP).

\section{References}

1. Abidin, RR. Parenting Stress Index. Psychological Assessment Resources; Odessa, FL: 1995.

2. Anderson, BJ.; Coyne, JC. "Miscarried helping" in famlies of children and adolescents with chronic diseases. In: Johnson, JH.; Johnson, SB., editors. Advances in child health psychology. University of Florida Press; Gainsville, FL: 1991.

3. Crombez G, Bijttebier P, Eccleston C, Mascagni T, Mertens G, Goubert L, Verstraeten K. The child version of the pain catastrophizing scale (PCS-C): a preliminary validation. Pain. 2003; 104(3):639646. [PubMed: 12927636]

4. D’Zurilla, TJ.; Nezu, AM. Problem solving therapy: A social competence approach to clinical intervention. 2nd edition. Springer Publishing; New York: 1999.

5. D'Zurilla, TJ.; Nezu, AM. Problem solving therapy: A positive approach to clinical intervention. 3rd edition. pringer Publishing Company, LLC; New York: 2007. 
6. D’Zurilla, TJ.; Nezu, AM.; Maydeu-Olivares, A. Manual for the Social Problem-olving InventoryRevised. Multi-Health Systems; North Tonawanda, NY: 2002.

7. Eccleston C, Crombez G, Scotford A, Clinch J, Connell H. Adolescent chronic pain: Patterns and predictors of emotional distress in adolescents with chronic pain and their parents. Pain. 2004; 108(3):221-229. [PubMed: 15030941]

8. Eccleston C, Jordan A, McCracken LM, Sleed M, Connell H, Clinch J. The Bath Adolescent Pain Questionnaire (BAPQ): development and preliminary psychometric evaluation of an instrument to assess the impact of chronic pain on adolescents. Pain. 2005; 118(1-2):263-270. [PubMed: 16202524]

9. Eccleston C, Palermo TM, Fisher E, Law E. Psychological interventions for parents of children and adolescents with chronic illness. Cochrane Database of Systematic Reviews. 2012; 8:CD009660. doi: 10.1002/14651858.CD009660.pub2. [PubMed: 22895990]

10. Fales JL, Essner BS, Harris MA, Palermo TM. When helping hurts: miscarried helping in families of youth with chronic pain. Journal of Pediatric Psychology. in press.

11. Fisher E, Heathcote L, Palermo T, Williams A, Lau J, Eccleston C. Systematic review and metaanalysis: Psychological therapies for children with chronic pain. Journal of Pediatric Psychology. in press.

12. Goubert L, Eccleston C, Vervoort T, Jordan A, Crombez G. Parental catastrophizing about their child's pain. The parent version of the Pain Catastrophizing Scale (PCS-P): a preliminary validation. Pain. 2006; 123(3):254-263. [PubMed: 16644128]

13. Grant JS, Elliott TR, Weaver M, Bartolucci AA, Giger JN. Telephone intervention with family caregivers of stroke survivors after rehabilitation. Stroke. 2002; 33(8):2060-2065. [PubMed: 12154263]

14. Harris MA, Antal H, Oelbaum R, Buckloh LM, White NH, Wysocki T. Good intentions gone awry: assessing parental "miscarried helping" in diabetes. Families, Systems \& Health. 2008; 26(4):393-403.

15. Haskett ME, Ahern LS, Ward CS, Allaire JC. Factor structure and validity of the parenting stress index-short form. Journal of Clinical Child \& Adolescent Psychology. 2006; 35(2):302-312. [PubMed: 16597226]

16. Huguet A, Miro J. The severity of chronic pediatric pain: an epidemiological study. Journal of Pain. 2008; 9(3):226-236. [PubMed: 18088558]

17. Hunfeld JA, Perquin CW, Hazebroek-Kampschreur AA, Passchier J, van Suijlekom-Smit LW, van der Wouden JC. Physically unexplained chronic pain and its impact on children and their families: the mother's perception. Psychology and Psychotherapy. 2002; 75(Pt 3):251-260. [PubMed: 12396752]

18. Jordan A, Eccleston C, Crombez G. Parental functioning in the context of adolescent chronic pain: a review of previously used measures. Journal of Pediatric Psychology. 2008; 33(6):640-659. [PubMed: 18203775]

19. Jordan A, Eccleston C, McCracken LM, Connell H, Clinch J. The Bath Adolescent Pain--Parental Impact Questionnaire (BAP-PIQ): development and preliminary psychometric evaluation of an instrument to assess the impact of parenting an adolescent with chronic pain. Pain. 2008; 137(3): 478-487. [PubMed: 18035497]

20. Jordan AL, Eccleston C, Osborn M. Being a parent of the adolescent with complex chronic pain: an interpretative phenomenological analysis. European Journal of Pain. 2007; 11(1):49-56. [PubMed: 16458550]

21. Kashikar-Zuck S, Swain NF, Jones BA, Graham TB. Efficacy of cognitive-behavioral intervention for juvenile primary fibromyalgia syndrome. Journal of Rheumatology. 2005; 32(8):1594-1602. [PubMed: 16078340]

22. Kazdin AE, Whitley MK. Treatment of parental stress to enhance therapeutic change among children referred for aggressive and antisocial behavior.[see comment]. Journal of Consulting \& Clinical Psychology. 2003; 71(3):504-515. [PubMed: 12795574]

23. Kelley ML, Heffer R, Gresham F, Elliot S. Development of a modified treatment evaluation inventory. Journal of Psychopathology and Behavioral Assessment. 1989; 11:235-247. 
24. Law E, Fisher E, Fales J, Noel M, Eccleston C. Systematic review and meta-analysis: Parent and family based interventions for children and adolescents with chronic medical conditions. Journal of Pediatric Psychology. in press.

25. Lewandowski AS, Palermo TM, Stinson J, Handley S, Chambers CT. Systematic review of family functioning in families of children and adolescents with chronic pain. Journal of Pain. 2010; 11(11):1027-1038. doi: S1526-5900(10)00487-6 [pii]10.1016/j.jpain.2010.04.005. [PubMed: 21055709]

26. Logan DE, Scharff L. Relationships between family and parent characteristics and functional abilities in children with recurrent pain syndromes: an investigation of moderating effects on the pathway from pain to disability. Journal of Pediatric Psychology. 2005; 30(8):698-707. [PubMed: 16093517]

27. McNair, DM.; Lorr, M.; Droppleman, LF. Manual for the Profile of Mood States. EdITS; San Diego, CA: 1992.

28. Morley S, Williams AC, Black S. A confirmatory factor analysis of the Beck Depression Inventory in chronic pain. Pain. 2002; 99(1-2):289-298. [PubMed: 12237207]

29. Palermo TM, Chambers CT. Parent and family factors in pediatric chronic pain and disability: An integrative approach. Pain. 2005; 119(1-3):1-4. [PubMed: 16298492]

30. Palermo TM, Eccleston C. Parents of children and adolescents with chronic pain. Pain. 2009; 146(1-2):15-17. [PubMed: 19482426]

31. Palermo TM, Putnam J, Armstrong G, Daily S. Adolescent autonomy and family functioning are associated with headache-related disability. Clinical Journal of Pain. 2007; 23(5):458-465. [PubMed: 17515745]

32. Rivera PA, Elliott TR, Berry JW, Grant JS. Problem-solving training for family caregivers of persons with traumatic brain injuries: a randomized controlled trial. Archives of Physical Medicine and Rehabilitation. 2008; 89(5):931-941. [PubMed: 18452743]

33. Ross CK, Lavigne JV, Hayford JR, Berry SL, Sinacore JM, Pachman LM. Psychological factors affecting reported pain in juvenile rheumatoid arthritis. Journal of Pediatric Psychology. 1993; 18(5):561-573. [PubMed: 8295080]

34. Sahler OJ, Fairclough DL, Phipps S, Mulhern RK, Dolgin MJ, Noll RB, Butler RW. Using problem-solving skills training to reduce negative affectivity in mothers of children with newly diagnosed cancer: report of a multisite randomized trial. Journal of Consulting and Clinical Psychology. 2005; 73(2):272-283. [PubMed: 15796635]

35. Sahler OJ, Varni JW, Fairclough DL, Butler RW, Noll RB, Dolgin MJ, Mulhern RK. Problemsolving skills training for mothers of children with newly diagnosed cancer: a randomized trial Journal of Developmental \& Behavioral Pediatrics. 2002; 23(2):77-86. [PubMed: 11943969]

36. Sanders MR, Shepherd RW, Cleghorn G, Woolford H. The treatment of recurrent abdominal pain in children: a controlled comparison of cognitive-behavioral family intervention and standard pediatric care. Journal of Consulting and Clinical Psychology. 1994; 62(2):306-314. [PubMed: 8201068]

37. Seid M, Varni JW, Gidwani P, Gelhard LR, Slymen DJ. Problem-solving skills training for vulnerable families of children with persistent asthma: Report of a randomized trial on healthrelated quality of life outcomes. Journal of Pediatric Psychology. 2010; 35:1133-1143. [PubMed: 20061311]

38. Stanford EA, Chambers CT, Biesanz JC, Chen E. The frequency, trajectories and predictors of adolescent recurrent pain: A population-based approach. Pain. 2008; 138(1):11-21. [PubMed: 18093737]

39. Wade SL, Carey J, Wolfe CR. An online family intervention to reduce parental distress following pediatric brain injury. J Consult Clin Psychol. 2006; 74(3):445-454. [PubMed: 16822102] 


\section{Table 1}

\section{Sample demographics}

\begin{tabular}{lccc}
\hline & Mean/\% & SD & Range \\
\hline Child Age (years) & 14.5 & 2.74 & $9-16$ \\
Child Sex (\% female) & $66.7 \%$ & & \\
Child Race & & & \\
$\quad$ Caucasian & $100 \%$ & & \\
Child Usual Pain Intensity (0-10 NRS) & 7.6 & 1.34 & $6-9$ \\
Pain Location & & & \\
$\quad$ Abdominal & $17 \%$ & & \\
$\quad$ Musculoskeletal & $66 \%$ & & \\
$\quad$ Head & $17 \%$ & & \\
Caregiver (\% mother) & $100 \%$ & & \\
Marital Status & & \\
$\quad$ Married & $60 \%$ & \\
$\quad$ Divorced & $40 \%$ & \\
Family income & & \\
Less than \$49,999 & $20 \%$ & \\
\$50,000-99,999 & $40 \%$ & \\
More than \$100,000 & $40 \%$ & \\
Parent Education & & \\
Vocational School/Some College & $20 \%$ & \\
Completed College & $20 \%$ & \\
Graduate/Professional School & $60 \%$ & \\
\end{tabular}


Table 2

Description of PSST treatment goals and content by session

\begin{tabular}{|c|c|}
\hline Session & Goals and Content \\
\hline 1 & $\begin{array}{l}\text { The primary goal of the first session is to establish a positive therapeutic } \\
\text { relationship through demonstrating understanding of the child's pain problem and impact on parent and family. A secondary goal is } \\
\text { to present PSST rationale and overall treatment program goals. } \\
\text { - Therapist obtains story of child's pain, and focuses on establishing rapport } \\
\text { - Therapist provides general explanation of the goals of the training, the problem solving model of stress, and the } \\
\text { structure of the treatment program and ground rules }\end{array}$ \\
\hline 2 & $\begin{array}{l}\text { The primary goal of the second session is to provide a core introduction to the } \\
\text { problem-solving process for the caregiver to acquire instruction in learned } \\
\text { optimism and problem orientation. } \\
\text { - Therapist provides instruction in how to identify connections between automatic thoughts and feelings, and how to } \\
\text { generate and use positive self statements }\end{array}$ \\
\hline 3 & $\begin{array}{l}\text { By the end of the third training session, a complete introduction is made to all of } \\
\text { the problem-solving steps. The primary goal is for the caregiver to acquire } \\
\text { instruction in Identifying Problems, Defining Options, Evaluating Options, and Act. } \\
\text { - } \quad \text { Therapist collaborates with caregivers to identify problems to solve, and to break up complex problems into } \\
\text { manageable parts } \\
\text { - Therapist introduces each problem solving step by working through a problem identified by caregiver }\end{array}$ \\
\hline $4-6$ & $\begin{array}{l}\text { The goal of these treatment sessions is to describe solution verification and } \\
\text { continue to practice the problem solving steps working through the same problem } \\
\text { or new problems } \\
\text { - } \\
\text { - Therapist reviews solution verification and discusses caregiver's level of satisfaction with the solution } \\
\text { Therapist helps caregiver to select alternative solution if caregiver not satisfied with outcome or to identify new } \\
\text { problem to be worked on if satisfied with first outcome }\end{array}$ \\
\hline $6-8$ & $\begin{array}{l}\text { The goals for these sessions are to discuss progress and plan for therapy } \\
\text { termination and to review skill maintenance and relapse prevention strategies. } \\
\text { - } \quad \text { Therapist emphasizes using emotional distress as a cue for initiating problem solving strategies in the future. } \\
\text { - } \quad \text { Therapist emphasizes the importance of skilled persistence and self-efficacy for solving problems on own. }\end{array}$ \\
\hline
\end{tabular}


Table 3

Pre- and post-treatment mean scores on parent and child outcome measures

\begin{tabular}{lccc}
\hline \multicolumn{1}{c}{ Scale } & Range & $\begin{array}{c}\text { Pre-treatment } \\
\text { M (SD) }\end{array}$ & $\begin{array}{c}\text { Post-treatment } \\
\text { M (SD) }\end{array}$ \\
\hline Parent-reported Outcomes & & & \\
Social Problem Solving Inventory-Revised (SPSI-R) & $0-208$ & $100.6(18.3)$ & $113.3(5.3)$ \\
Beck Depression Inventory (BDI-II) & $0-63$ & $14.0(8.5)$ & $3.0(3.6)$ \\
POMS (Total Mood Disturbance) & $-32-200$ & $54.0(11.0)$ & $48.8(7.2)$ \\
Parenting Stress Index-Short Form (PSI-SF) & $36-180$ & $90.8(8.0)$ & $55.0(45.8)$ \\
Helping for Health Index (HHI) & $0-60$ & $37.0(8.9)$ & $28.8(6.5)$ \\
Pain Catastrophizing Scale for Parents (PCS-P) & $0-52$ & $36.8(9.9)$ & $25.5(7.1)$ \\
BAP-PIQ: Parental behavior & $0-55$ & $20.6(6.8)$ & $16.3(3.3)$ \\
Treatment Evaluation Inventory-Short Form & $9-45$ & --- & $36.5(2.5)$ \\
Child-reported Outcomes & & & \\
BAPQ - Physical functioning & $0-36$ & $11.0(2.0)$ & $6.8(2.2)$ \\
BAPQ - Depression & $0-24$ & $15.8(5.0)$ & $11.5(4.0)$ \\
\hline
\end{tabular}

